\title{
Nonholonomic motion planning for a free-falling cat using spline approximation
}

\author{
GE XinSheng $^{1 *} \&$ GUO ZhengXiong ${ }^{2}$ \\ ${ }^{1}$ College of Mechanical \& Electrical Engineering, Beijing Information Science \& Technology University, Beijing 100192, China; \\ ${ }^{2}$ Institute of Mechanics, Chinese Academy of Sciences, Beijing 100190, China
}

Received October 26, 2011; accepted February 29, 2012; published online September 14, 2012

\begin{abstract}
An optimal motion planning of a free-falling cat based on the spline approximation is investigated. Nonholonomicity arises in a free-falling cat subjected to nonintegrable velocity constraints or nonintegrable conservation laws. The equation of dynamics of a free-falling cat is obtained by using the model of two symmetric rigid bodies. The control of the system can be converted to the motion planning problem for a driftless system. A cost function is used to incorporate the final errors and control energy. The motion planning is to determine control inputs to minimize the cost function and is formulated as an infinite dimensional optimal control problem. By using the control parameterization, the infinite dimensional optimal control problem can be transformed to a finite dimensional one. The particle swarm optimization (PSO) algorithm with the cubic spline approximation is proposed to solve the finite dimension optimal control problem. The cubic spline approximation is introduced to realize the control parameterization. The resulting controls are smooth and the initial and terminal values of the control inputs are zeros, so they can be easily generated by experiment. Simulations are also performed for the nonholonomic motion planning of a free-falling cat. Simulated experimental results show that the proposed algorithm is more effective than the Newtoian algorithm.
\end{abstract}

falling cat, nonholonomic constraint, motion planning, spline approximation, particle swarm optimization

PACS number(s): 02.06.-x, 45.04.-f, 45.20.dc, 45.50.Jf

Citation: Ge X S, Guo Z X. Nonholonomic motion planning for a free-falling cat using spline approximation. Sci China-Phys Mech Astron, 2012, 55: 2100-2105, doi: 10.1007/s11433-012-4891-6

\section{Introduction}

It is well known that a cat, when released from an upside down configuration starting from rest, is able to land on its feet. At the end of the 19th century, people began to try to explain this interesting phenomenon. Guyou and Marey [1] firstly explained from classical mechanics that the angular momentum of a falling cat is conserved. McDonald [2] also represents this problem from a physiological point of view. He believed a cat firstly contracts its front feet, then protracts the front feet while rotating its front body. Meanwhile,

*Corresponding author (email: gebim@vip.sina.com) its rear body also experiences a rotation. According to the conservation law of angular momentum, the rotation angle of the front body is larger than that of the rear body in the opposite direction. This theory satisfied the principle of mechanics. However, in free-falling cat experiments, we hardly find any obvious protract-contract motion of a cat's feet. Лойцянский et al. [3] presented another explanation, where the rapid rotation of the cat's tail makes its body turn over in the opposite direction. However, this conjecture is not valid either. Experiments show that a cat without tail can also finish the rotating motion. Kane and Scher [4] proposed the dynamical explanation of the phenomenon that a free-falling cat usually lands on its feet. They assumed the cat's turning motion with its waist as the top point using the 
model of two symmetric rigid bodies. Based on this model, a set of governing equations was established and the general characteristic of the turning motion was obtained. Further numerical analysis showed this model was in good agreement with experimental results. For the more general condition of two unsymmetrical rigid body's turning motion, a set of dynamics equations was set up by Liu [1].

Recently, with the development of manned spacecrafts and exploratory researches of human turning motion under zero-gravity conditions, the research of a free-falling cat has become a significant topic. Due to the non-integrable angle velocity, the first integration of the equation of a cat's rotation is an equation with nonholonomic constrains, and it is a special nonlinear system. In this equation, the dimension of generalized coordinates is larger than that of the control input. Brockett et al. [5] first suggested a systematic research on the optimal control problem of the driftless nonholonomic system. Using control objective functions to construct Lagrangian functions, they reached conclusions under optimal input of sinusoidal function and elliptical function respectively. Murray and Sastry extended Brockett's conclusion to the control of nonholonomic chain system under sinusoidal input [6]. A similar motion planning method was also given by Reyhanoglu and Mukherjee, which used Stokes theorem and Taylor series expansion to analyze the dynamic model of the nonholonomic system $[7,8]$. For motion planning problems of nonholonomic control systems, various numerical methods were achieved by some investigators. Fernandes et al. formulated the nonholonomic motion planning problem as an optimal control problem, and developed a simple algorithm for a coupled rigid body system using ideas from Ritz's Approximation Theory [9]. Yih and Ro proposed the algorithms of near optimal motion planning using multipoint shooting and quasi Newtonian method for nonholonomic systems $[10,11]$. Duleba and Sasiadek discussed a modification of the Newton algorithm applied to nonholonomic motion planning with energy optimization [12]. The Lyapunov control method for solving motion planning was proposed by Tsuchiya et al. [13]. In this method, the control input was obtained by multiplying the gradient vector of the Lyapunov function by a tensor. Ge et al. studied an optimal control of a free-falling cat using the Newtonian algorithm by Fourier approximation [14].

In this paper, the motion of free-falling cat is formulated through a double rigid body model which can represent the front and rear half of its body. The motion equation of a free-falling cat is established based on multibody dynamic and conservation of angular momentum. When the total angular momentum is zero, the attitude motion equations of a free-falling cat take on nonholonomic constraint. The control of a free-falling cat can be converted to the motion planning problem without drift. By using the control parameterization, the infinite dimensional optimal control problem can be transformed to a finite dimensional one. The particle swarm optimization (PSO) algorithm with the cubic spline approximation is proposed to resolve the optimal control of a free-falling cat. Finally, the algorithm is tested through simulation. The simulation results indicate that the algorithm is an effective approach to deal with a free-falling cat.

\section{The attitude motion equation of a free-falling cat}

To simplify the free-falling cat motion model, the body of a cat is taken as two symmetric rigid bodies $B_{1}$ and $B_{2}$ which are joined at $O$. Assume the rigid bodies are torsion free and only bending exists when the cat bends its spine shown in Figure 1 . The coordinate systems $O-X_{i} Y_{i} Z_{i}(i=1,2)$ are prescribed as follows: $O X_{i}$ is the centroid axis of the rigid bodies pointing from $O$ to the head of the cat $(i=1)$ or the tail of the cat $(i=2), O Z_{i}$ points to the abdomen of the cat.

The coordinate system $\mathrm{O}-\mathrm{X}_{2} \mathrm{Y}_{2} \mathrm{Z}_{2}$ is obtained by firstly rotating about axis $O X_{1}$ through angle $\psi$ to obtain $O-X_{1}^{*} Y_{1}^{*} Z_{1}^{*}$ as shown in Figure 2, then rotating about axis $O Y_{1}^{*}$ through angle $\vartheta$ to obtain $O-X_{1}^{\#} Y_{1}^{\#} Z_{1}^{\#}$, and finally rotating about axis $O X_{1}^{\#}$ through angle $\phi$ to obtain $O-X_{2} Y_{2} Z_{2}$. After getting $O-X_{2} Y_{2} Z_{2}$, we construct a new coordinate system $O-X^{*} Y^{*} Z^{*}$, in which $O X^{*}$ and $O Z^{*}$ are along the bisector of $\angle X_{1}^{*} O X_{1}^{\#}$ and $\angle Z_{1}^{*} O Z_{1}^{\#}$ separately, and $O Y^{*}$ is coincident to $O Y_{1}^{*}$ and $O Y_{1}^{\#}$. The angle $\gamma$, which equals $\vartheta / 2$, is the angularity between the front half (or rear half) spine and $O X^{*} . X^{*} O Z^{*}$ is the spine-curving plane. $\psi$ denotes the position of the plane in the cat's body.

The angular velocity $\omega^{\prime}$ of $B_{2}$ with respect to $B_{1}$ is obtained by projection on the $O-X^{*} Y^{*} Z^{*}$ coordinate system as:

$$
\omega^{\prime}=(\dot{\psi}+\dot{\phi}) \cos \gamma i^{*}+2 \dot{\gamma} j^{*}+(\dot{\psi}-\dot{\phi}) \sin \gamma k^{*} .
$$

According to the torsion free condition, the velocity component along axis $O X^{*}$ must be zero, and then we obtain the relationship $\dot{\phi}=-\dot{\psi}$. Since the initial condition is also torsion free, we get $\phi=-\psi$ by employing integration. Considering the relation between angle $\phi$ and $\psi$, eq. (1) can be simplified as:

$$
\omega^{\prime}=2\left(\dot{\gamma} j^{*}+\dot{\psi} \sin \gamma k^{*}\right) .
$$

After bending the spine, the center of mass of the cat $O_{c}$ locates on axis $O Z^{*}$. If we move the origin from $O$ to $O_{c}$, the axis $O_{c} X_{0}$ keeps a steady horizontal direction during the observation of a free-falling cat. A new coordinates $O_{c}-X Y Z$ is built, in which $O_{c} X$ is coincident to $O_{c} X_{0}$, and the axis $O_{c} Z$ goes upward and vertically to the ground. During the process of free falling, the inertia forces in $O_{c}-X Y Z$ are balanced with gravity. When we consider rotating about the 


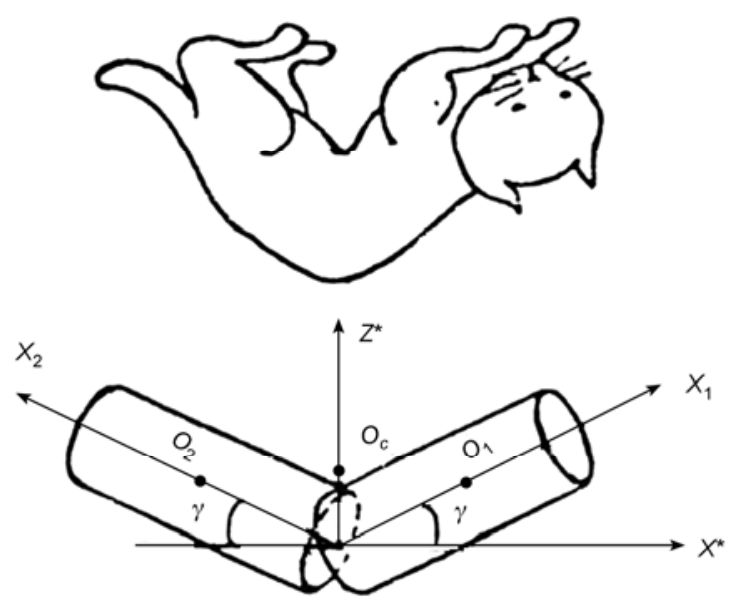

Figure 1 A free-falling cat model.

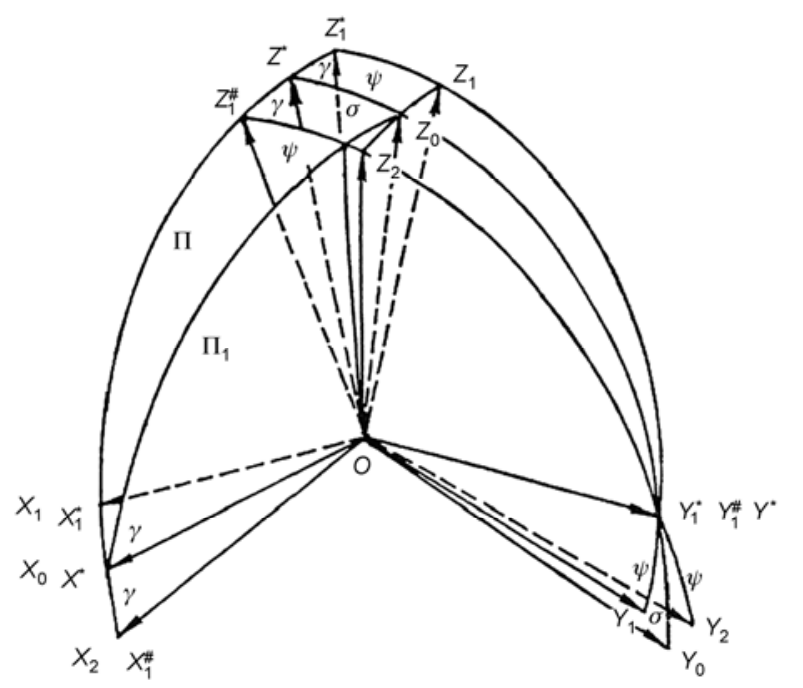

Figure 2 Attitude angle transform.

center of mass, coordinates $O_{c}-X Y Z$ can be taken as the inertial reference frame. Set vertical plane $X O_{c} Z$ as $\Pi_{0}$, and let $\phi$ be the clockwise angle from plane $\Pi_{0}$ to $\Pi_{1}$. The purpose of rotation motion of the cat is to make its abdomen from facing upward to downward, namely, the angle $\phi$ from 0 to $\pi$.

The angular velocities of $B_{i}(i=1,2)$ with respect to $O-X^{*} Y^{*} Z^{*}$ reference frame are

$$
\begin{aligned}
& \omega_{1}^{*}=-\dot{\psi} \cos \gamma i^{*}-\dot{\gamma} j^{*}-\dot{\psi} \sin \gamma k^{*}, \\
& \omega_{2}^{*}=\omega_{1}^{*}+\omega^{\prime}=-\dot{\psi} \cos \gamma i^{*}+\dot{\gamma} j^{*}+\dot{\psi} \sin \gamma k^{*} .
\end{aligned}
$$

Let $A, B, C, m$ and $a$ be the central inertia moments, the mass and the distance between the centroid and $O$ of $B_{i}$ $(i=1,2)$ respectively. The moment of momentum $\boldsymbol{H}_{i}$ of $B_{i}$ with respect to $O_{c}$ could be computed. The vector $\boldsymbol{H}_{i}$ can be decomposed into components with respect to $O-X_{i} Y_{i} Z_{i}(i=1$, 2) coordinate systems [1]:

$$
\left\{\begin{array}{l}
H_{1 x} \\
H_{1 y} \\
H_{1 z}
\end{array}\right\}=\left\{\begin{array}{c}
H_{2 x} \\
-H_{2 y} \\
-H_{2 z}
\end{array}\right\}=\left[\begin{array}{ccc}
A_{c} & -F_{c} & -E_{c} \\
-F_{c} & B_{c} & -D_{c} \\
-E_{c} & -D_{c} & C_{c}
\end{array}\right]\left\{\begin{array}{l}
p \\
q \\
r
\end{array}\right\},
$$

where

$$
\begin{aligned}
& A_{c}=A+m a^{2} \cos ^{2} \gamma \sin ^{2} \gamma, \\
& B_{c}=B+m a^{2} \cos ^{2} \gamma\left(\cos ^{2} \gamma+\sin ^{2} \gamma \cos ^{2} \psi\right) \text {, } \\
& C_{c}=C+m a^{2} \cos ^{2} \gamma\left(\cos ^{2} \gamma+\sin ^{2} \gamma \sin ^{2} \psi\right) \text {, } \\
& D_{c}=m a^{2} \cos ^{2} \gamma \sin ^{2} \gamma \cos \psi \sin \psi \text {, } \\
& E_{c}=m a^{2} \cos ^{3} \gamma \sin \gamma \cos \psi \text {, } \\
& F_{c}=m a^{2} \cos ^{3} \gamma \sin \gamma \sin \psi, \\
& p=\left[\left(\begin{array}{llll}
\dot{\psi} \cos \gamma+\dot{\gamma} & \sin \gamma \cos \psi \sin \psi
\end{array}\right)\right. \\
& \left./\left(1-\sin ^{2} \gamma \cos ^{2} \psi\right)-\phi\right] \cos \gamma-\dot{\psi},
\end{aligned}
$$

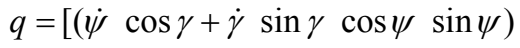

$$
\begin{aligned}
& \left./\left(1-\sin ^{2} \gamma \cos ^{2} \psi\right)-\phi\right] \sin \gamma \sin \psi-\dot{\gamma} \cos \psi, \\
& r=[\phi-(\dot{\psi} \cos \gamma+\dot{\gamma} \sin \gamma \cos \psi \sin \psi) \\
& \left./\left(1-\sin ^{2} \gamma \cos ^{2} \psi\right)\right] \sin \gamma \cos \psi-\dot{\gamma} \sin \psi .
\end{aligned}
$$

The sum of $\boldsymbol{H}_{1}$ and $\boldsymbol{H}_{2}$ is the total moment of momentum of the cat with respect to $O_{c}$. After transformation to the $O-X^{*} Y^{*} Z^{*}$ coordinate system, the component of the sum along axis $O X^{*}$ is

$$
\begin{aligned}
H= & -2\left\{\left[A \cos ^{2} \gamma+\left(B \sin ^{2} \psi+C \cos ^{2} \psi\right) \sin ^{2} \gamma\right]\right. \\
& {[\phi-(\dot{\psi} \cos \gamma+\dot{\gamma} \sin \gamma \cos \psi \sin \psi)} \\
& \left./\left(1-\sin ^{2} \gamma \cos ^{2} \psi\right)\right] \\
& +A \dot{\psi} \cos \gamma+(B-C) \dot{\gamma} \sin \gamma \cos \psi \sin \psi\} i^{*} .
\end{aligned}
$$

During the process of falling a cat, the moment with respect to centroid is zero. Since the angular momentum $\boldsymbol{H}$ is conservative, the assumption of invariance of the direction of axis $O_{c} X^{*}$ or $O_{c} X$ is proved to be correct. By considering $\boldsymbol{H} \equiv$ 0 , we can obtain the motion equation from eq. (7) given by

$$
\phi=\frac{\left\{\dot{\psi} \cos \gamma \sin \gamma\left[\sigma+(1-\varepsilon) \cos ^{2} \psi\right]+\dot{\gamma} \cos \psi \sin \psi\left(1-\varepsilon+\sigma \sin ^{2} \gamma\right)\right\} \sin \gamma}{\left(1-\sin ^{2} \gamma \cos ^{2} \psi\right)\left[1+\left(\sigma-\varepsilon \cos ^{2} \psi\right) \sin ^{2} \gamma\right]},
$$

where $\sigma=(B-A) / A, \quad \varepsilon=(B-C) / A$ are parameters associated with the mass of cat. Eq. (8) is the nonholonomic attitude motion equation of a free-falling cat.

\section{Nonholonomic motion planning by spline approximation method}

Consider the system configuration variable $\boldsymbol{x}=(\psi, \gamma, \phi)^{\mathrm{T}} \in$ 
$\boldsymbol{R}^{3}$, and notice that $\boldsymbol{u} \in \boldsymbol{R}^{2}$ is the control input with $u_{1}=\dot{\psi}$ and $u_{2}=\dot{\gamma}$. Introducing the above expressions in eq. (8), one has

$$
\dot{\boldsymbol{x}}=\boldsymbol{G}(\boldsymbol{x}) \boldsymbol{u}, \boldsymbol{x}_{0}, \boldsymbol{x}_{f} \in \boldsymbol{R}^{3}
$$

$$
G(x)=\left[\begin{array}{c}
1 \\
0 \\
\frac{\cos \gamma \sin ^{2} \gamma\left[\sigma+(1-\varepsilon) \cos ^{2} \psi\right]}{\left(1-\sin ^{2} \gamma \cos ^{2} \psi\right)\left[1+\left(\sigma-\varepsilon \cos ^{2} \psi\right) \sin ^{2} \gamma\right]}
\end{array}\right.
$$

The nonholonomic motion planning problem is to find control inputs steering the system (9) from a given initial configuration $x_{0}$ to a final state $x_{\mathrm{f}}$ in time $T$ for the minimum control energy. Assuming that the system is controllable [15], there must exist a solution $u \in L_{2}([0, T])$ for the problem. Here, $\boldsymbol{L}_{2}([0, T])$ denotes the Hilbert space of measurable vector functions of the form $\boldsymbol{u}(t), t \in[0, T]$. Based on the law of minimal energy control, dissipated energy of each rotation joint of a free-falling cat is chosen to minimize the cost function. It can be described as follows:

$$
\left\{\begin{array}{l}
\min f(\boldsymbol{u})=\int_{0}^{\boldsymbol{T}}\langle\boldsymbol{u}, \boldsymbol{u}\rangle \mathrm{d} t \\
\text { subject to } \boldsymbol{x}(T)=\boldsymbol{x}_{\boldsymbol{f}}
\end{array}\right.
$$

Thus, the problem of nonholonomic motion planning is equivalent to an infinite dimensional optimization problem. We can use the calculus of variations to find the necessary optimality conditions for the above nonlinear optimal control problem. Since the system is nonlinear, the optimal control depends on the solution of the nonlinear multi-point boundary value problem $[10,11]$. Numerical schemes such as multi-point shooting method and quasi-linearization method can be used to solve the boundary value problem. However, one needs to have a close solution to the optimum point in order to ensure the convergence of these numerical algorithms. Also, since only necessary conditions are satisfied, it is not guaranteed that the solution is a minimum point to the optimization problem. For complicated underactuated systems like the rigid spacecraft with two wheels, it is a very difficult task to compute the optimal conditions.

The problems mentioned above can be overcome by using the control parameterization method. Using the parameterization of the control variables, one can transform the infinite dimensional optimization problem to a finite dimensional problem. In this paper, each control input is approximated by the cubic spline control. The cubic spline interpolation is a most common piecewise-polynomial approximation. It is not only continuously differentiable on the interval, but also has a continuous second derivative. Given a set of nodes $0=t_{0}<t_{1}<\cdots<t_{N}=T$, and the values of control inputs in those nodes $\lambda=\left[u_{0}, u_{1}, \cdots, u_{N}\right]^{\mathrm{T}}$, a natural cubic spline approximation can be constructed [16]

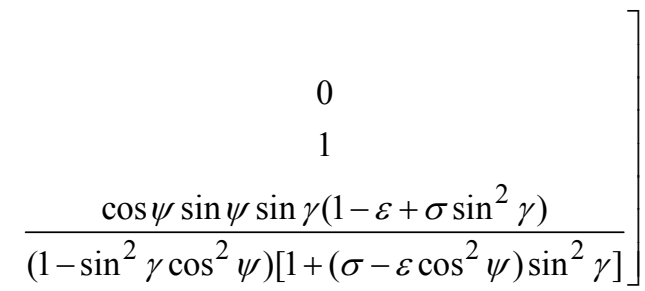

$$
u(t)=S(\lambda, t), \quad t \in[0, T] .
$$

Taking $\alpha$ as a new control vector and applying the theory of penalty function, substituting eq. (11) to eq. (10), one can obtain

$$
F(\lambda, \alpha)=\int_{0}^{\mathrm{T}}[S(\lambda, t)]^{2} \mathrm{~d} t+\alpha\left\|f(\lambda)-x_{f}\right\|^{2},
$$

where $\alpha$ is a penalty parameter. Solution $\lambda$ can be obtained through minimizing the unconstrained optimization problem given by eq. (10). The limit of $\lambda$ is the solution of the problem given by eq. (10) as $\alpha \rightarrow \infty$.

Because the cubic spline approximation has a continuous second derivative, and the initial value of control input can be predefined as arbitrary value, the resulting control inputs are easily generated by motors.

\section{The optimal control with particle swarm op- timization}

In the particle swarm algorithm, the trajectory of each individual in the search space is adjusted by dynamically altering the velocity of each particle, according to its own flying experience and the flying experience of other particles in the search space. The particles have a tendency to fly toward better search areas over the course of a search process. The PSO algorithm is given as follows [17]:

$$
\begin{gathered}
V_{t+1}=w V_{t}+c_{1} \operatorname{rand}()\left(P_{i}-X_{t}\right)+c_{2} \operatorname{rand}()\left(P_{g}-X_{t}\right), \\
X_{t+1}=X_{t}+V_{t+1},
\end{gathered}
$$

where $\operatorname{rand}()$ generates uniformly distributed random figures in the range $[0,1] . w$ is called the inertia weight and is less than $1, V_{t}$ and $X_{t}$ represent the speed and the position of the particle at time $t, P_{i}$ refers to the best position found by the particle, and $P_{g}$ refers to the global best position found by the whole particle swarm. $c_{1}$ and $c_{2}$ are constants known as acceleration coefficients.

The solution of unconstrained optimization problem (12) can be resolved by the PSO algorithm. Using the updating eqs. (13) and (14), the pseudo code for nonholonomic mo- 
tion planning is as follows:

Begin

initialize the population and the penalty parameter

for $(k=1$ to $M)$

while(the error of the trajectory end-point $<\varepsilon$ )

for $(i=1$ to $N)$

calculate the fitness $F\left(\boldsymbol{X}_{i}\right)$, update $\boldsymbol{P}_{i}$ and $\boldsymbol{P}_{g}$

$\boldsymbol{V}_{t+1}=w \boldsymbol{V}_{t}+c_{1} \operatorname{rand}()\left(\boldsymbol{P}_{i}-\boldsymbol{X}_{t}\right)+c_{2} \operatorname{rand}()\left(\boldsymbol{P}_{g}-\boldsymbol{X}_{t}\right)$

$\boldsymbol{X}_{t+1}=\boldsymbol{X}_{t}+\boldsymbol{V}_{t+1}$

increase $i$

end while

$$
r=c r
$$

increase $k$

end.

where, $M$ is the changing times of penalty parameter $r, N$ is the number of particles in the PSO algorithm, and $c>1$ is a scale factor. The penalty parameter $r$ increases with the running of the optimal process. The parameters, $\lambda$, in eq. (12) correspond to $X_{i}$ in eqs. (13) and (14).

\section{Numerical simulation}

Assume that during the process of a cat's free falling, only its spine bends and there is no rotation between the front and rear body. Also assume that the cat bends its spine forward to all the directions in turn and keeps angle $\gamma$ constant. When the front body of cat finishes a circle motion, the whole body of cat turns $p$ in the reverse direction, i.e. when the angle $\psi$ changes from 0 to $2 \pi$, the angle $\phi$ changes from 0 to $\pi$. From the experimental data, $\sigma \approx 3,|\varepsilon| \ll 1$. In the simulation experiment, let $\sigma=3, \varepsilon=0.01$, parameters $N=20$, $\xi=120 \mathrm{diag}\left[307.82 .5\right.$ ], $\zeta=194.85, e=10^{-6}$, the time interval of falling $t=1 \mathrm{~s}$. The prescribed time space in simulation computation is $0.05 \mathrm{~s}$.

We denote the initial position and the end position of a free-falling cat as:

$$
\boldsymbol{x}_{0}=\left(\begin{array}{lll}
0 & \pi / 6 & 0
\end{array}\right)^{\mathrm{T}}, \boldsymbol{x}_{\boldsymbol{f}}=(2 \pi-\pi / 6 \pi)^{\mathrm{T}} .
$$

The control inputs are approximated by cubic spline interpolation. Six parameters and 10 parameters are selected to construct the cubic spline function and the initial values and final values are predefined as zero. The number of particles in the PSO algorithm is set as 15 .

In case 1 , the optimal parameterization vectors, $\lambda_{1}=(0$, $7.6304,6.9315,6.1626,7.9481,0)^{\mathrm{T}}$ for $u_{1}$ and $\lambda_{2}=(0,0.9617$, $-0.2293,-1.4009,-3.9462,0)^{\mathrm{T}}$ for $u_{2}$, are obtained by the PSO algorithm. The energy of the optimal trajectory equals $J=46.6951$. The simulation results are shown in Figures 3 and 4 . Figure 3 shows plots of the optimal control inputs for the middle joint of the double rigid body. Figure 4 shows the attitude optimal trajectories of the cat during its falling. In case 2 , the optimal parameterization vectors, $\lambda_{1}=(0$,
$7.3156,6.2829,7.3269,7.3731,6.3557,6.2621,5.9847$, $7.0186,0)^{\mathrm{T}}$ for $u_{1}$ and $\lambda_{2}=(0,1.5054,0.1261,-0.4861$, $-1.7109,-4.4987,-3.4949,-1.6012,0.1889,0)^{\mathrm{T}}$ for $u_{2}$, are obtained by the PSO algorithm. The energy of the optimal trajectory equals $J=45.7667$. The simulation results are shown in Figures 5 and 6. Figure 5 shows plots of the optimal control inputs for the middle joint of the double rigid body. Figure 6 shows the attitude optimal trajectories of the cat during its falling. The two ends of the curves are individually the initial point and the landing point. Compared with the Fourier series approximation, $J=29.7$ units energy is saved by using the spline approximation that both of the initial values and final values of the control are zero. From Figures 4 and 6, it can be seen that the control inputs start at zero values and land on zero values also. It is obvious the cat experiences a steady rotation. There is no detour behavior in the turndown motion. We can see the bending angle has a small amplitude variation in Figures 4 and 6 . These simulation results are very inosculate to the experimental record.

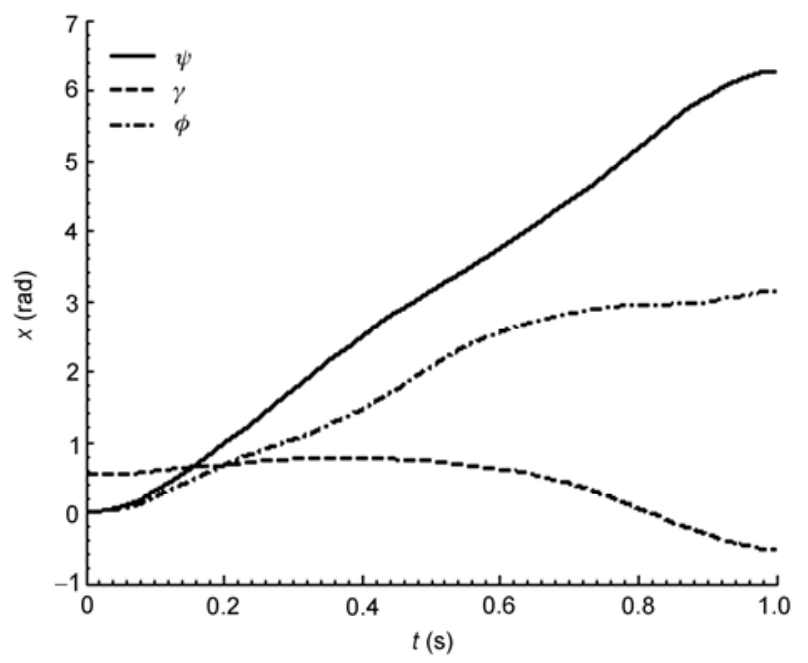

Figure 3 Optimal trajectory for the attitude angle $(n=4)$.

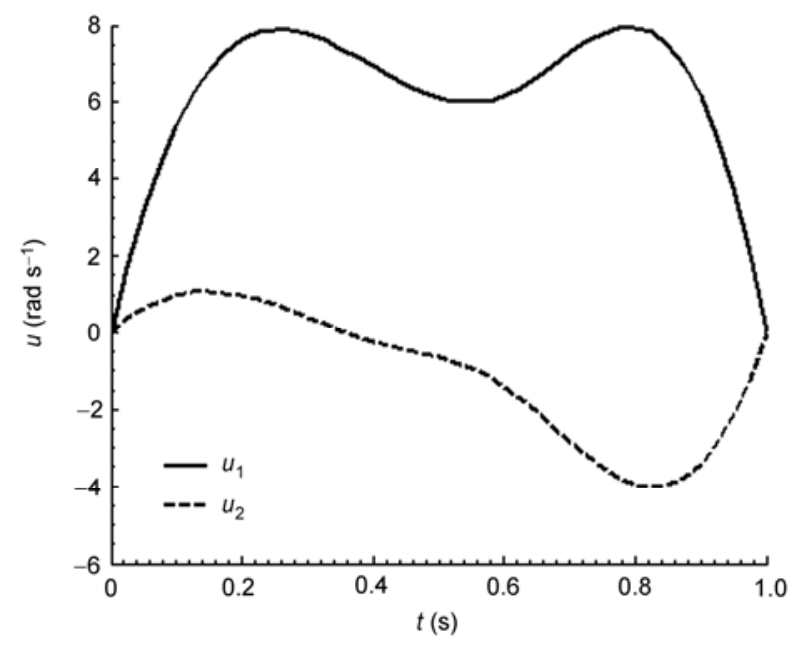

Figure 4 The optimum control input for a free-falling cat $(n=4)$. 


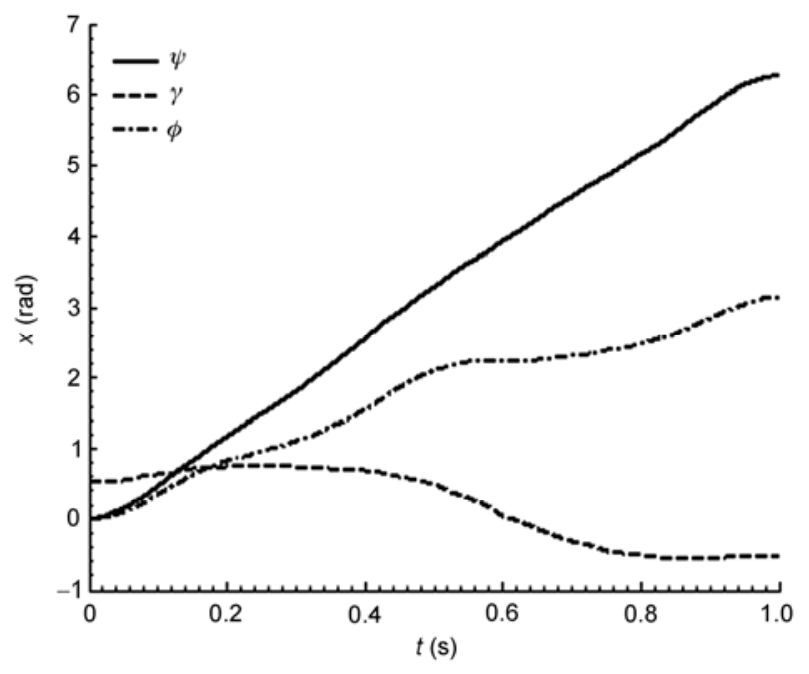

Figure 5 Optimal trajectory for the attitude angle $(n=8)$.

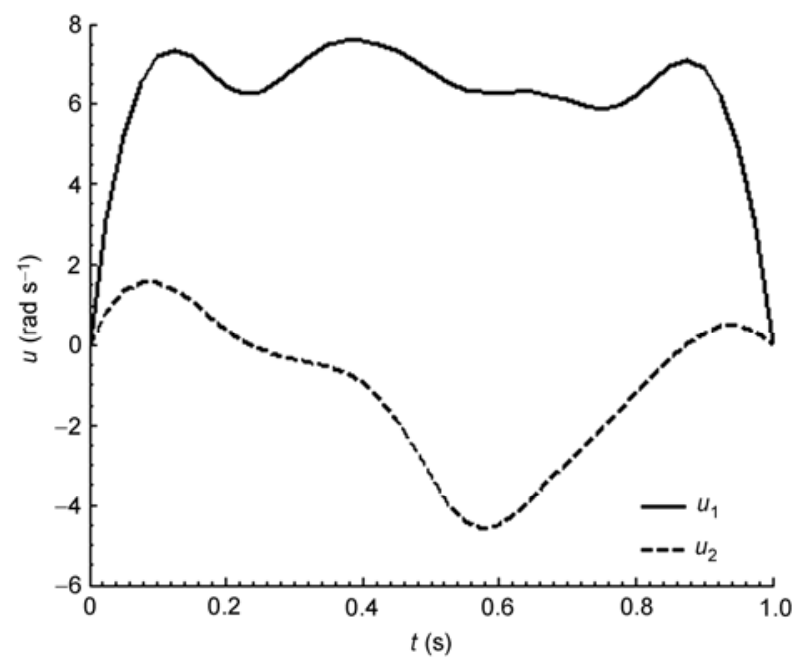

Figure 6 The optimum control input for a free-falling cat $(n=8)$.

\section{Conclusion}

From the modeling of a free-falling cat and numerical analysis, we get the following conclusions. Firstly, the nonlinear control problem of a free-falling cat can be transformed to a nonholonomic motion planning problem of a driftless system. It can be solved effectively by spline approximation, which implements the attitude planning of a free-falling cat and the optimal of control input. During the simulation computation, the spline approximation method shows fast convergence speed and good accuracy.

Secondly, in the motion planning method that control inputs are parameterized Fourier series and wavelet approximation $[14,18]$, smooth control trajectories are obtained. However, the start and final values of control inputs are non-zero values and they are not easy to implement with typical servomotors. Comparing with Fourier approximation method, one can find that excellent performance has been obtained by the proposed method in this paper. The result- ing control inputs are smooth and start at zero values and land on zero values also.

Thirdly, the Newtonian algorithm for the nonholonomic motion planning is sensitive to the initial value. For different initial values, different local optima can be obtained. The PSO is insensitive to the initial values, and stands a good chance of finding the global optimum. A PSO algorithm is proposed to resolve the nonholonomic motion planning, which is parameterized by spline approximation.

This work was supported by the National Natural Science Foundation of China (Grant No. 11072038) and the Municipal Key Programs of Natural Science Foundation of Beijing, China (Grant No. KZ201110772039).

1 Marey M. Des mouvements que certains animaux exécutent pour retomber sur leurs pieds, lorsqu'ils sont précipités d'un lieu élevé. Comp Rend Acad Sci Paris, 1984, (119): 714-717; Guyon M. Note relative à la communication de M. Marey. Comp Rend Acad Sci Paris, 1984, (119): 717-718; Liu Y Z. On the turning motion of a freefalling cat (in Chinese). Acta Mech Sin, 1982, 14(4): 388-393

2 McDonald D A. How dose a falling cat turn over. Am J Physiol, 1955, 129: 34-35

3 Лойцянский Л Г, Лурье А И. Курс Теоретичес Коймеханики. Москва: Гостехиздат, 1954

4 Kane T R, Scher M P. A dynamical explanation of the falling cat phenomenon. Int J Solids Struct, 1969, 5(7): 663-670

5 Brockett R W, Dai L. Nonholonomic kinematics and the role of elliptic functions in constructive controllability. Li Z, Canny J F, eds. Nonholonomic Motion Planning. Norwell, Massachusetts: Kluwer, 1993. 1-22

6 Murray R M, Sastry S S. Nonholonomic motion planning: Steering using sinusoids. IEEE Trans Automatic Control, 1993, 38(5): 700716

7 Reyhanoglu M. A general nonholonomic motion planning strategy for Caplygin systems. In: Proceedings of the $33^{\text {rd }}$ IEEE Conference on Decision and Control. Florida, USA, 1994. 2964-2966

8 Mukherjee R, Anderson D P. A Surface integral approach to the motion planning of nonholonomic systems. ASME J Dyn Syst Measur Control, 1994, 116(9): 315-325

9 Fernandes C, Gurvits L, Li Z. Near-optimal nonholonomic motion planning for a system of coupled rigid bodies. IEEE Trans Automation Control, 1995, 39(3): 450-464

10 Yih C C, Ro P I. Near-optimal motion planning for nonholonomic systems using multi- point shooting method. In: Proc. IEEE Conf. Robotics and Automation, Minneapolis, M N, 1996. 2943-2948

11 Yih C C, Ro P I. Near-optimal motion planning for nonholonomic systems with state/input constraints via quasi-Newton method. In: Proc. IEEE Conf. Robotics and Automation, Albuquerque, New Mexico, 1997. 1430-1435

12 Duleba I, Sasiadek J Z. Nonholonomic motion planning based on newton algorithm with energy optimization. IEEE Trans Control Syst Tech, 2003, 11(3): 355-363

13 Tsuchiya K, Urakubo T, Tsujita K. Motion control of a nonholonomic systems based on the Lyapunov control method. J Guidance Control Dyn, 2002, 25(2): 285-290

14 Ge X S, Chen L Q. Optimal control of nonholonomic motion planning for a free-falling cat. Appl Math Mech, 2007, 28(5) : 601-607

15 Murray R M, Li Z, Sastry S S. A Mathematical Introduction to Robotic Manipulation. CRC Press, 1994

16 Burden R L, Faires J D. Numerical Analysis. 7th ed. Beijing: Higher Education Press and Thomson Learning, 2001

17 Joshi M C, Moudgalya K N. Optimization Theory and Practices. Harrow, U. K.: ASI Ltd., 2004

18 Ge X S, Chen L Q, Liu Y Z. Nonlinear attitude control of an underactuated spacecraft using wavelet approximate method. Prog Nat Sci, 2006, 17(8): 868-873 